\title{
Ex vivo and in vitro production of pro-inflammatory cytokines in Blau syndrome
}

\author{
P. Galozzi', O. Negm², 3, E. Greco'1, N. Alkhattabi², A. Gava1, P. Sfriso', \\ L. Fairclough'2, I. Todd', P. Tighe'2, L. Punzi' \\ ${ }^{1}$ Rheumatology Unit, Department of Medicine - DIMED, University of Padova, Italy; \\ ${ }^{2}$ School of Life Sciences, Department of Immunology, University of Nottingham, Nottingham, United Kingdom; \\ ${ }^{3}$ Medical Microbiology and Immunology Departement, Faculty of Medicine, Mansoura University, Egypt
}

\section{SUMMARY}

The objective was to study both ex vivo and in vitro secretion of pro-inflammatory cytokines in patients affected by Blau syndrome (BS) and carrying p.E383K mutation in the CARD15/NOD2 gene associated with the disease.

For ex vivo studies, peripheral blood mononuclear cells (PBMCs), serum from three patients and healthy controls have been collected. PBMCs have been cultured in the presence or absence of inflammatory enhancers, such as lipopolysaccharide (LPS) and muramyl dipeptide (MDP). The levels of interleukin (IL)-1 $\beta$, IL-6, IL-8, tumor necrosis factor (TNF)- $\alpha$ and interferon (IFN)- $\gamma$ were assayed by either immunoassay or array-based system. For in vitro studies, different constructs were created cloning human wild-type and p.E383K-mutated NOD2 cDNA into the expression vector pCMV-Tag2c. HEK293 cell lines were stably transfected, cultured with or without MDP and IL-8 level was assayed in their surnatants. Statistical analysis in both studies was performed using non-parametric tests.

Both ex vivo and in vitro studies have not identified a significant increase in secretion of the analyzed proinflammatory cytokines. p.E383K-mutated NOD2 transfected cells express low level of IL-8. The ex vivo basal level results from both serum and PBMCs surnatants present similar levels of IL- $1 \beta$, IL-6, TNF- $\alpha$ and IFN- $\gamma$ in patients and controls. The presence of the stimulant agents (LPS and MDP), either individual or paired, does not lead to significant increases in all cytokines concentrations in patients compared to controls.

Taken together, the ex vivo and in vitro data suggest that there is not a primary mediation of IL-1 $\beta$ and other pro-inflammatory cytokines in BS patients carrying p.E383K.

Key words: Pro-inflammatory cytokines, Blau syndrome, Autoinflammatory disease, Interleukin-1ß.

Reumatismo, 2014; 66 (4): 277-284

\section{INTRODUCTION}

lau syndrome (BS, MIM \#186580) is a rare autoinflammatory granulomatous disorder, dominantly inherited, described in 1985 as a chronic inflammatory syndrome characterized by granulomatous dermatitis, symmetric arthritis and recurrent uveitis (1). The eye involvement is the most relevant morbidity of BS, presenting in particular as granulomatous uveitis that can evolve into cataract and band keratopathy. Skin manifestations are described as papulonodular, tender, brownish rash or multiple, firm, subcutaneous plaques only apparent on palpation. The most frequent joint involvement in BS is inflammatory arthritis, with moderate redness, warmth and tenderness, associated with synovial cysts and tenosynovitis with a chronic evolution (2). Due to the rarity and variable expressivity of BS, no studies on the optimal treatment have been made yet. Low-dose glucocorticoids are generally acceptable at the quiescent stage (3), although biologic anti-cytokine agents (infliximab and anakinra) may be a promising therapeutic approach in refractory cases (4).

The gene responsible for BS was identified in 2001 in the caspase recruitment domain 15 gene (CARD15/NOD2), mapped in the chromosomal region 16q12.1-13 (5). Since then, several cohorts of BS patients have been genotyped, leading to identification
Conference presentation: L SIR Congress, 2013.
Corresponding author: Galozzi Paola UOC di Reumatologia Dipartimento di Medicina - DIMED Via Giustiniani, 2 - 35128 Padova, Italy E-mail: paola.galozzi@unipd.it 
of different CARD15/NOD2 mutations from distinct ethnic origins. Until now, 17 missense substitutions have been observed associated with BS. Among those, the mutations affecting the highly evolutionary conserved Arginine residue at codon 334 could be counted as mutational hot spot (6-8). In 2005, our group has identified the mutation p.E383K in an Italian family affected by BS (9). Mutations in CARD15/ NOD2 have also been associated with a higher risk of Crohn's disease (MIM \#266600), a multifactorial inflammatory bowel disorder characterized by transmural granulomatous inflammation of the gastrointestinal tract (10). CARD15/NOD2 gene encodes a multi-domain protein of 1040 amino acids, called nucleotide oligomerization domain 2 (NOD2), presenting a three-domain structure composed of two $\mathrm{N}$-terminal effector regions CARD, a central activator NACHT, and nine C-terminal pathogen-sensing leucine-rich repeats (LRRs) (11). NOD2 is a member of the NOD-like receptor family (NLR), highly expressed in monocytes and granulocytes. There, it plays an important role in innate immune defence as intracellular receptor for bacterial products, i.e. muramyl dipeptide (MDP) (12). Upon recognizing MDP, NOD2 triggers host defence response by inducing nuclear factor (NF)- $\kappa \mathrm{B}$ activation through a cascade of phosphorylations. Once activated (NF)- $\mathrm{BB}$, it translocates to the nucleus and starts the transcription of pro-inflammatory genes (13). The BSassociated mutations are reported to be related in vitro with elevated basal and MDP-induced NF- $\kappa \mathrm{B}$ activity, supporting a gain-of-function theory and suggesting a spontaneous release of pro-inflammatory cytokines, firstly interleukin (IL)-1 $\beta$ (14). Despite the fact that BS is genetically wellcharacterized, few studies in the literature have analyzed the cytokines profile in BS. Elevated serum levels of IL-1 $\beta$, IL- 6 and tumor necrosis factor (TNF)- $\alpha$ in BS patients have been reported by Arosteguji et al. (15). However, different other studies could not confirm an increase in proinflammatory cytokines levels (16-18). Based on this controversy, we analyzed both ex vivo and in vitro the cytokines profile in BS patients carrying p.E383K mutation.

\section{MATERIALS AND METHODS}

\section{Subjects}

Three subjects with the classical manifestation of BS and 5 gender- and agematched healthy controls were included in this study. The three BS patients belong to the same Italian family (Fig. 1), the only one carrying the p.E383K mutation (9). The proband (II4), now 61 years old, was referred to our unit in 1984 and diagnosed as BS on the basis of the described criteria (19). Main clinical signs were widespread papules and firm subcutaneous nodules at the upper and lower limbs, severe bilateral uveitis, and symmetrical arthritis of the small joints of the hands. At the time of this study, the proband presented arthralgia of the lower limbs treated with methylprednisolone $4 \mathrm{mg} / \mathrm{day}$ and granulomatous uveitis evolved into cataract and band keratopathy. The daughter (III2), now 36 years old, showed similar, although less severe, skin manifestations, uveitis and arthritis. Her clinical state at the time of this study was good, while she was assuming methylprednisolone $4 \mathrm{mg} /$ day. The grand-daughter (IV1), now 5 years old, has not developed any clinical BS manifestation yet.

\section{Collection of serum and stimulated pe- ripheral blood mononuclear cell}

After informed consent, serum and blood samples were obtained from BS patients and healthy controls. Peripheral blood mononuclear cells (PBMCs) were isolated by Ficoll-density gradient separation and seeded at a density of $10^{6}$ cell $/ \mathrm{mL}$ in RPMI media containing 1\% Glutamine and $10 \%$ FCS. Cells were cultured for $24 \mathrm{~h}$ in an atmosphere of $95 / 5 \%$ air $/ \mathrm{CO}_{2}$ at $37^{\circ} \mathrm{C}$ either without stimulation or in the presence of muramyl dipeptide $[10 \mu \mathrm{g} / \mathrm{mL}]$ (SigmaAldrich, St. Louis, MO, USA), purified lipopolysaccharide from E.Coli 055:B5 [100 $\mathrm{ng} / \mathrm{mL}$ ] (Sigma-Aldrich) or a combination of both. 


\section{Construction of NOD2-encoding plas- mids}

Expressing plasmid pCMV-Tag2c encoding amino-terminally FLAG-tagged NOD2 wild-type (kindly provided by Dr T. Kufer) was the starting point for obtaining mutated NOD2 expressing plasmids. p.E383K mutation was introduced by using QuikChangeXL site-directed mutagenesis kit (Stratagene). All constructs were subjected to full-length sequencing.

\section{Stable transfection into HEK293 cells and stimulation}

Human HEK293 cells were cultured in DMEM High glucose $90 \%$ medium (Life Technology, Carlsbad, CA, USA) supplemented with $0.1 \%$ Gentamycin, $10 \%$ FCS and $2 \%$ L-glutamine. Stable transfections were performed by using Lipofectamine ${ }^{\mathrm{TM}}$ 2000 (Life Technologies), according to the manufacturer's directions. After $48 \mathrm{~h}$ the transfected cell lines were selected adding $0.8 \mathrm{mg} / \mathrm{mL} \mathrm{G} 418$ (Life Technology). A control cell line was generated by transfecting an empty mock vector.

Transfected HEK293 cells were cultured for 7 and $24 \mathrm{~h}$ either with or without muramyl dipeptide stimulation $[10 \mu \mathrm{g} / \mathrm{mL}]$ (Sigma-Aldrich).

\section{Cytokine analysis}

Human IL-1 $\beta$, IL-6, IL- 8 , TNF- $\alpha$ and IFN- $\gamma$ in PBMCs supernatants were measured by immunoassays using commercial kits (IMMULITE, Siemens, Berlin, Germany) according to the manufacturer's instructions.

In serum samples, the cytokines were assayed using an array-based system. Briefly, capture antibodies dilutions $[100 \mu \mathrm{g} / \mathrm{mL}]$ were prepared in a 384-well plate and spotted on poly-l-lysine slides by MicroGrid II robot (BioRobotics Inc., Ramsey, MN, USA).

After creating separated wells with proper slide holders, the printed slides were blocked with I-Block solution (Applied Biosystem, Waltham, MA, USA) and incubated for $1 \mathrm{~h}$ with serum samples, along with a cocktail of antibodies standards to enable quantification. A mix of biotinyl-

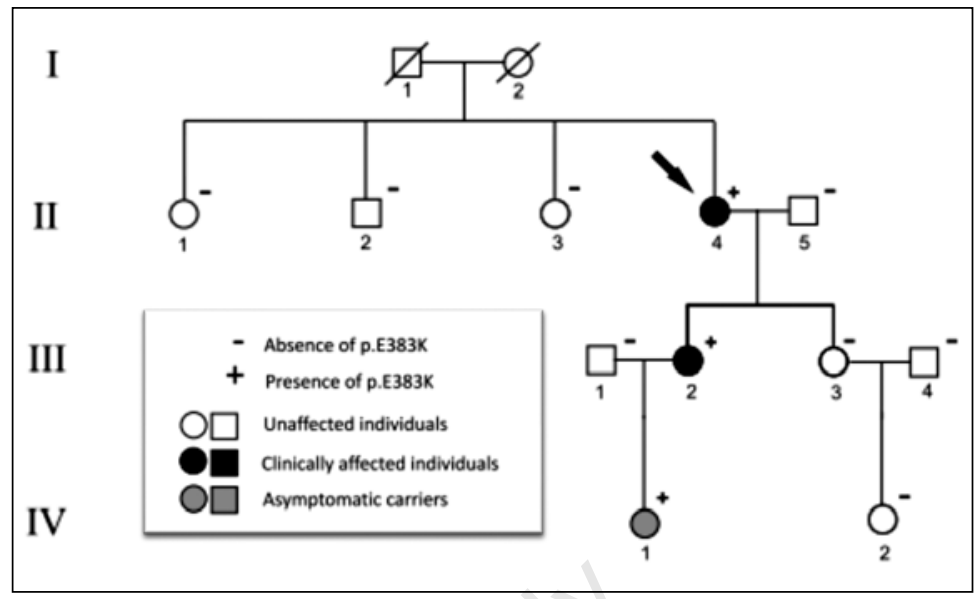

Figure 1 - Pedigree structure of the proband and her family. Filledin symbols indicate clinically affected family members, both proband (subject II-4) and her daughter (subject III-2). Shaded symbol represents proband's granddaughter (subject IV-1), an asymptomatic carrier of the p.E383K mutation. Absence (-) or presence $(+)$ of p.E383K mutation is indicated.

ated detection antibodies was added then to each block and incubated for $1 \mathrm{~h}$. Serum samples need an amplification step to improve the signal sensitivity (TSA, Bio-rad Lab., Hercules, CA, USA). Streptavidinconjugated cy 5 at $1: 1000$ is finally incubated for $15 \mathrm{~min}$, then the slides are dried by centrifugation and scanned at $635 \mathrm{~nm}$ with Axon 4200AL scanner.

In surnatants of NOD2-transfected HEK293 cells, IL-8 was measured by the same array-based system described above, without the amplification step.

All experiments were made in duplicate.

\section{Data processing and statistics}

The immunoassay data were used for statistical comparisons between the means of data obtained from experimental groups and those from control groups, using analysis of variance with Fisher's post hoc (GraphPad Prism 5).

Cy5 fluorescence, gained from the microarray system, was instead quantified using GenePix Pro-6 software (Axon Instrument, Union City, CA, USA), subtracted from the background and used for computing average and standard deviation. The concentrations of each cytokine were calculated based on a standard curve of known 
amounts of standard cytokines. Betweengroup comparisons were performed with non-parametric tests (Kruskal-Wallis and Mann-Whitney) (GraphPad Prism 5). Statistical significance was defined as twosided $\mathrm{P}$-value $<0.05$.

\section{RESULTS}

To define the biological effects of p.E383K BS-associated mutation, we examined both ex vivo and in vitro the ability of this mutation to produce cytokines in the presence or absence of activating stimuli.

\section{Ex vivo cytokines profile}

As presented in Figure 2a, no statistical differences in cytokine levels were detected between patients and controls, ex- cept for IL-8 that was secreted at higher titre $(\mathrm{P}<0.05)$ by healthy controls in the absence of stimulation. When stimulated with the TLR agonist [lipopolysaccharide (LPS)], BS PBMCs released similar or statistically lower levels of cytokines compared to healthy controls, such as for IL-6 ( $\mathrm{P}<0.05)$ (Fig. 2b).

Despite these results, the cytokines levels raised significantly in patients after LPS stimulation, as expected (Fig. 3a). IL6 , IL-8 and TNF- $\alpha$ concentrations were highly released after LPS stimulation $(\mathrm{P}<0.005, \mathrm{P}<0.005$ and $\mathrm{P}<0.05$ respectively), with the exception of IL- $1 \beta$ and IFN- $\gamma$.

Even the NOD2 agonist (MDP) did not stimulate a high cytokine release in patients compared to healthy controls. A significant decrease, indeed, was seen

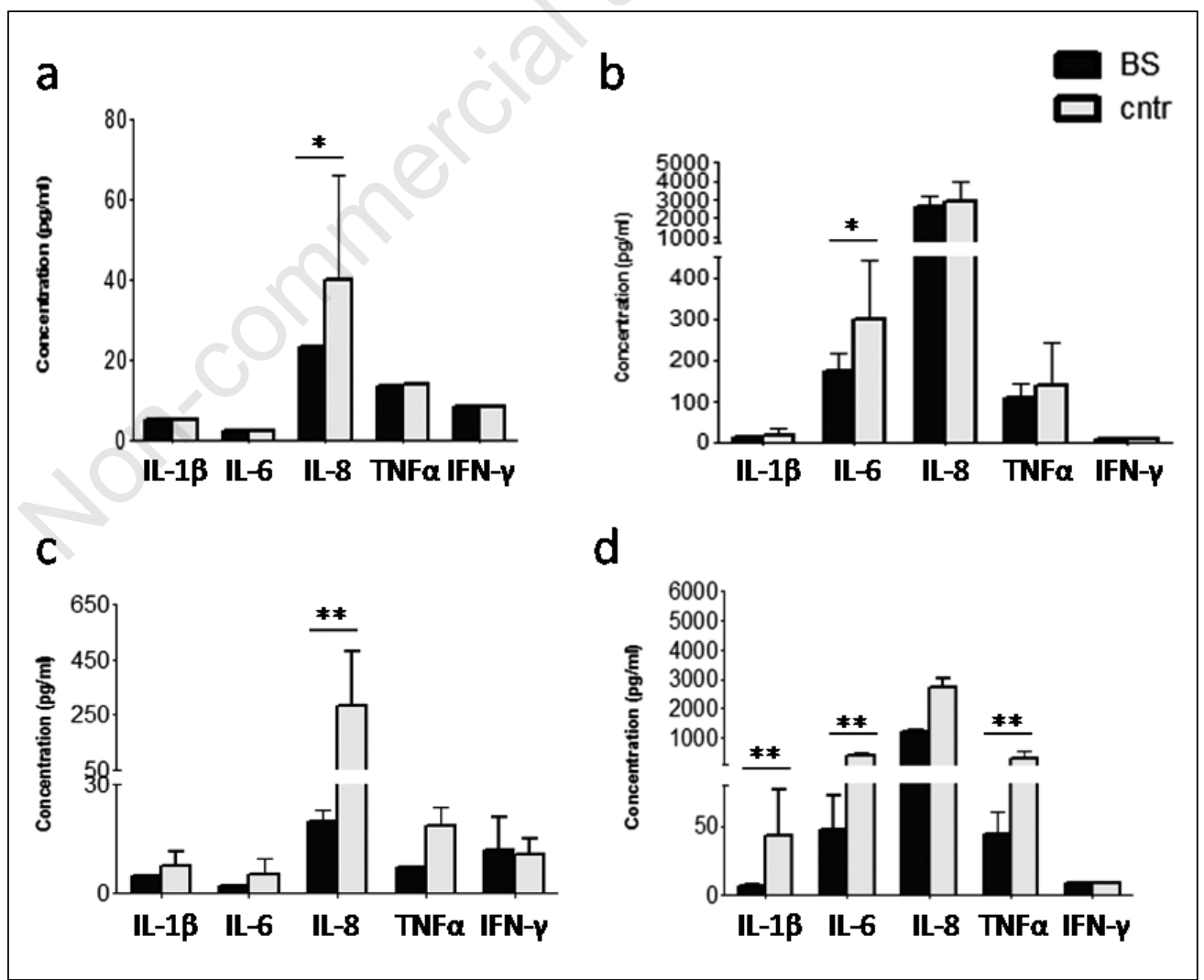

Figure 2 - Cytokines levels of peripheral blood mononuclear cells surnatants obtained from Blau syndrome patients (black) and healthy controls (grey) in the absence (a) or presence of lipopolysaccharide (b), muramyl dipeptide (c) and both stimulants (d) after $24 \mathrm{~h}$. The data shown are means of data from duplicate experiments and the error bars indicate standard deviation. ${ }^{\star} \mathrm{P}<0.05$; ${ }^{* \star} \mathrm{P}<0.005$. IL, interleukin; TNF- $\alpha$, tumor necrosis factor- $\alpha$; IFN- $\gamma$, interferon- $\gamma$. 


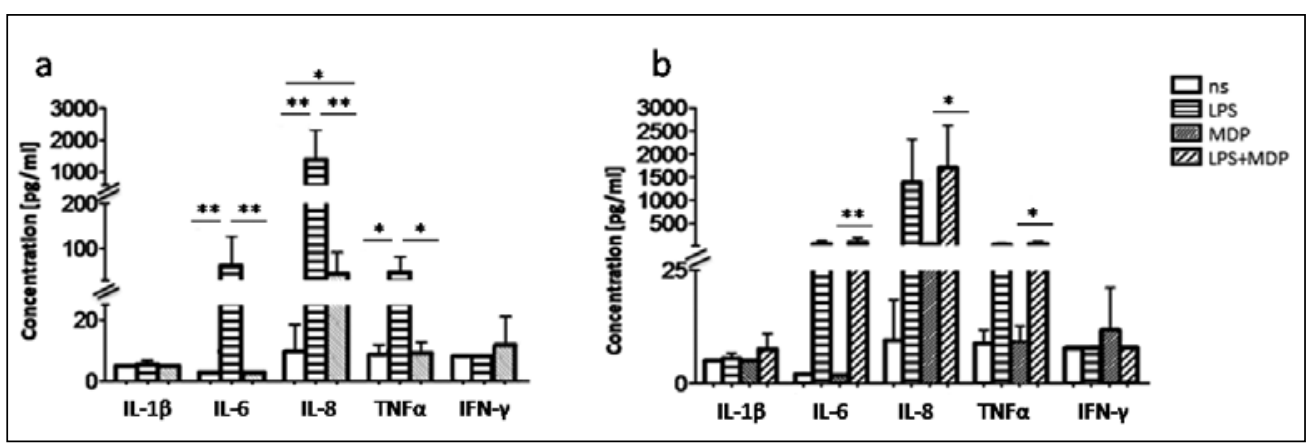

Figure 3 - Cytokines levels in peripheral blood mononuclear cells surnatants obtained from Blau syndrome patients after $24 \mathrm{~h}$. a) Comparison between individual stimuli and basal level; b) Comparison between double stimulation and muramyl dipeptide (MDP) stimulation. The data shown are means of data from duplicate experiments and the error bars indicate standard deviation. ${ }^{\star} \mathrm{P}<0.05$; ${ }^{* \star} \mathrm{P}<0.005$. ns, not significant; LPS, lipopolysaccharide; IL, interleukin; TNF- $\alpha$, tumor necrosis factor- $\alpha$; IFN- $\gamma$, interferon- $\gamma$.

in patients $(\mathrm{P}<0.005)$ for IL-8 (Fig. 2c). Evaluating only BS patients (Figure $3 \mathrm{a}$ ), it was notable that only IL-8 levels increased after MDP stimulation $(\mathrm{P}<0.05)$, while levels of all other cytokines were similar to the non-stimulated ones or statistically lower than LPS stimulated ones $(\mathrm{P}<0.005$ for IL- 6 and TNF- $\alpha)$. Moreover, this study failed to identify an increase in cytokine secretion in patients following double stimulation. IL- $1 \beta$, IL- 6 and TNF- $\alpha$ showed higher concentration in controls $(\mathrm{P}<0.005)$ (Fig. 2d), whereas, considering only patients, a synergistic effect was detected in IL-6, IL-8, and TNF- $\alpha$ production $(\mathrm{P}<0.005, \quad \mathrm{P}<0.05$, $\mathrm{P}<0.05$ respectively) (Fig. $3 \mathrm{~b}$ ).

The ex vivo results of serum samples at basal level (Fig. 4) reflect those presented above about PBMCs surnatants, i.e. no differences between patients and controls.

\section{In vitro cytokines profile}

Analyzing the data obtained after $7 \mathrm{~h}$ (Fig. 5a), we noted that in the absence of stimulation the levels of IL-8 in cells expressing wild-type NOD2 were statistically higher $(\mathrm{P}<0.05)$ than those obtained from p.E383K-mutated cells. When added MDP at the cell cultures, no differences were spotted between p.E383K and wild-type NOD2 transfected cells. A similar trend for both cell lines was observed for IL-8 secretion after $24 \mathrm{~h}$ (Fig. 5b).

\section{DISCUSSION}

The CARD15/NOD2 gene encodes the intracellular detector NOD2 that mediates $\mathrm{NF}-\kappa \mathrm{B}$ pathway activation in response to invasive bacterial infections. A distinct group of mutations in this gene causes, in heterozygous state, the rare autoinflammatory disease named Blau syndrome.

In 2005, our group has identified the mutation p.E383K in an Italian family affected by BS (9). This mutation is located in the

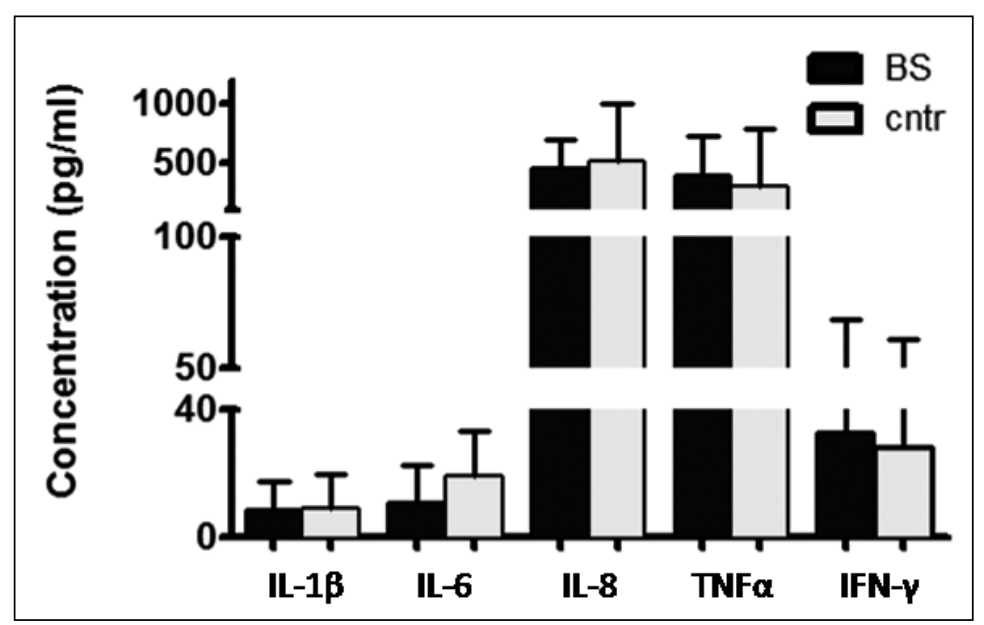

Figure 4 - Cytokines concentrations in serum obtained from Blau syndrome (BS) patients (black) and healthy controls (grey) at basal level. The bars indicate the means of data, whereas the error bars indicate standard deviation. No significant differences are observed. cntr, control; IL, interleukin; TNF- $\alpha$, tumor necrosis factor- $\alpha$; IFN- $\gamma$, interferon- $\gamma$. 


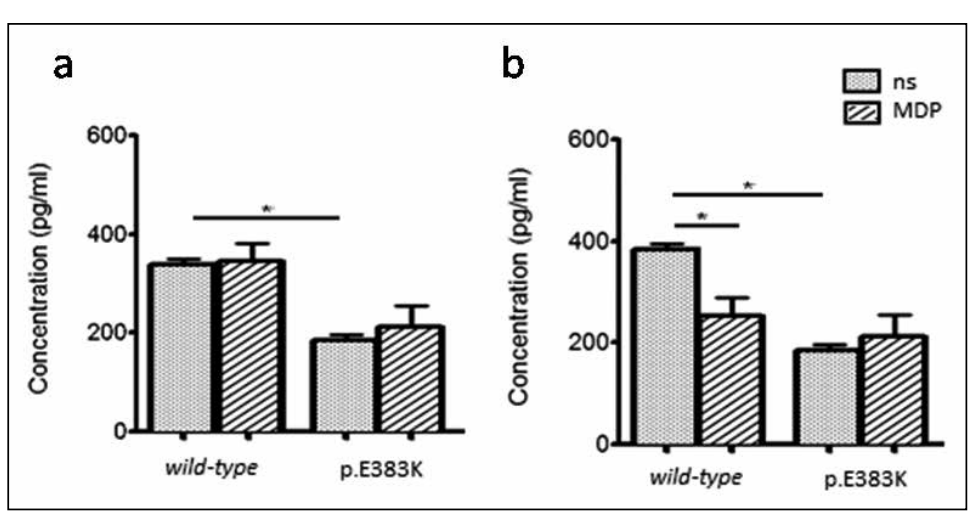

Figure 5 - Interleukin-8 levels of surnatants obtained from NOD2 transfected HEK293 cells stimulated or not with muramyl dipeptide (MDP) for 7 (a) and 24 (b) h. The data shown are means of data from duplicate experiments and the error bars indicate standard deviation. ${ }^{*} \mathrm{P}<0.05$. ns, not significant.

central domain and changes Glutamate 383 to Lysine. To date, no functional studies are presented in literature concerning this mutation. Its pathogenicity was supported by indirect pieces of evidence, such as its dominantly inheritance and its conservation among species.

The autoinflammatory nature of BS is related with its associated mutations. The gain of function hypothesis of BS mutations may suggest a spontaneous release of more cytokines, such as IL-1 $\beta$ (15). Moreover, several studies published in the literature have stated the importance of pro-inflammatory cytokines in driving the inflammation in different autoinflammatory conditions. Most of these studies underline the prominence of the IL- $1 \beta$ levels and its role in inflammasome activation and regulation, in particular on autoinflammatory diseases associated pyrin, NLRP12 and cryopyrin (NLRP3) proteins $(20,21)$. This has led to a breakthrough in therapy, with IL-1 inhibitors producing rapid and sustained amelioration of symptoms.

Moreover, also IL-6 is likely to be involved in the pathogenesis of some autoinflammatory diseases, due to high serum levels in periodic fevers and complex autoinflammatory disorders (Adult-Onset Still disease and Behçet disease) (22-24). Furthermore, IL-8 and TNF- $\alpha$ are known to increase in the presence of autoinflammatory conditions, such as TNF receptor-associated pe- riodic syndrome, Behçet and Adult-Onset Still diseases (25-27). However, recent works from the literature observed no evidence of excess pro-inflammatory cytokines such as IL- $1 \beta$ and TNF- $\alpha$ in PBMCs from BS patients compared to healthy controls $(16,17)$. In addition to these, Son and colleagues confirmed ex vivo that $\mathrm{BS}$ is not associated with increased basal levels of TNF- $\alpha$ (18).

We analyzed the up-regulation of pro-inflammatory cytokines production ex vivo and in vitro in p.E383K mutation carriers. Due to the small dimension of our BS family, we included individuals taking systemic anti-inflammatory medications in this study. However, the extracted PBMCs were less likely to be exposed to drugs during in vitro culturing, because cells underwent vigorous washing steps during the isolation process. This indicated that the observed cellular response was not influenced by patients treatment, but reflected the biologic response related to p.E383K mutation.

Our ex vivo data from patients PBMC and serum showed similar levels for IL-1 $\beta$, IL-6, IL-8, TNF- $\alpha$ and IFN- $\gamma$ production compared to healthy controls. The concentration values, instead, were generally higher in serum samples than PBMCs surnatants, as expected. LPS or MDP stimulation of PBMCs showed similar or lower levels of these cytokines than in healthy controls. Furthermore, no synergistic cytokine release was observed in patients compared to controls when muramyl dipeptide was used in combination with TLR agonist. In addition, our in vitro results did not show increased level of IL-8, both at basal and after MDP stimulation in p.E383Kmutated compared to wild-type NOD2.

Taken together, these findings suggest that an exaggerated NOD2 response leading to pro-inflammatory release is not a direct mechanism explaining the pathophysiology of BS. Moreover, the reduced response upon bacterial stimulation may limit the clearance by the host, contributing afterward to persistent inflammation and other clinical manifestations.

Focusing on IL-1 $\beta$, its slightly attenuated response in our BS subjects suggests that, 
in contrast with cryopyrinopathies and other hereditary periodic fever diseases, IL-1 $\beta$ do not primarily mediate the disease and IL-1 $\beta$ blocking therapy may not be an eligible treatment. In literature, some authors report the use of anakinra, IL-1 receptor antagonist, in some BS cases with variable results, in particular regarding ocular morbidity $(4,15)$.

Levels of the other cytokines analyzed were also not raised in p.E383K carriers. A case report of a BS patient, carrying a different mutation from the one we analyzed, in which IL-6 and IL- 8 were slightly elevated has been described (28). This may suggest a possible role of NOD2 in influencing the cytokines production, maybe related to differences in amino acid mutations.

The observed low level of TNF- $\alpha$ is consistent with the reported low clinical efficacy of TNF blocking therapies (infliximab and etanercept) in BS associated with recurrent infections $(4,16)$.

The similar levels of IFN- $\gamma$ between our BS patients and the controls need further investigations. IFN- $\gamma$ level would be expected to be elevated in BS patients due to its involvement in granulomas formation. Moreover, Rosenzweig et al. have demonstrated in a murine model a relation between NOD2 activation and IFN- $\gamma$ increased production within the eye (29), thus suggesting that dysregulation of IFN- $\gamma$ may occur in patients with BS and contribute to the granulomatous nature of the disease. Concerning our divergent result, a possible explanation may lie in the particular mutation we have analyzed or in the cells type we have collected.

Furthermore, the almost comparable cytokine levels between the affected subjects suggest that different disease states presented at the time of this study do not have visible effect on the results.

In summary, this study is the first that characterizes both ex vivo and in vitro cytokines profile in BS subjects carrying p.E383K mutation. Our results are consistent with those reported in literature by Martin et al. (16) and Son et al. (18), whilst their studies were based on p.R334W/Q mutated NOD2. Although our analysis suffers from a num- ber of limitations, in particular the small sample size, our data may be the starting point for studying the functional effects of p.E383K-mutated NOD2 in BS.

Acknowledgments: this work was supported, in part, by an unrestricted grant of Fondazione Ing. Aldo Gini, University of Padua, Italy.

\section{Acknowledgements}

The authors thanks Dr Kufer for providing the wild-type NOD2 plasmid and also Prof Rampazzo Alessandra and her collaborators Dr De Bortoli Marzia e Dr Dazzo Emanuela for their technical support in the mutagenesis process.

\section{REFERENCES}

1. Blau EB. Familial granulomatous arthritis, iritis, and rash. J Pediatr. 1985; 107: 689-93.

2. Sfriso P, Caso F, Tognon S, Galozzi P, Gava A, Punzi L. Blau syndrome, clinical and genetic aspects. Autoimmun Rev. 2012; 12: 44-51.

3. Latkany PA, Jabs DA, Smith JR, Rosenbaum JT, Tessler H, Schwab IR, et al. Multifocal choroiditis in patients with familial juvenile systemic granulomatosis. Am J Ophthalmol. 2002; 134: 897-904.

4. MilmanN, Ursin K, Rødevand E, Nielsen FC, Hansen TV. A novel mutation in the NOD2 gene associated with Blau syndrome: a Norwegian family with four affectedmembers. Scand J Rheumatol. 2009; 38: 190-7.

5. Tromp G, Kuivaniemi H, Raphael S, AlaKokko L, Christiano A, Considine E, et al. Genetic linkage of familial granulomatous inflammatory arthritis, skin rash, and uveitis to chromosome 16. Am J Hum Genet. 1996; 59: 1097-107.

6. Miceli-Richard C, Lesage S, Rybojad M, Prieur AM, Manouvrier-Hanu S, Häfner R, et al. CARD15 mutations in Blau syndrome. Nature Genet. 2001; 29: 19-20.

7. Rosé CD, Aróstegui JI, Martin TM, Espada G, Scalzi L, Yagüe J, et al. NOD2-associated pediatric granulomatous arthritis, an expanding phenotype: study of an international registry and a national cohort in Spain. Arthritis Rheum. 2009; 60: 1797-803.

8. Kanazawa N, Matsushima S, Kambe N, Tachibana T, Nagai S, Miyachi Y. Presence of a sporadic case of systemic granulomatosis syndrome with a CARD15 mutation. J Invest Dermatol. 2004; 122: 851-2.

9. vanDuist MM, Albrecht M, Podswiadek M, 
Giachino D, Lengauer T, Punzi L, et al. A new CARD15 mutation in Blau syndrome. Eur J Hum Genet. 2005; 13: 742-7.

10. Mekhjian HS, Switz DM, Melnyk CS, Rankin GB, Brooks RK. Clinical features and natural history of Crohn's disease. Gastroenterology. 1979; 77: 898.

11. Chen G, Shaw MH, Kim YG, Nuñez G. NODlike receptors: role in innate immunity and inflammatory disease. Annu Rev Pathol. 2009; 4: 365-98.

12. Inohara N, Ogura Y, Fontalba A, Gutierrez O, Pons F, Crespo J, et al. Host recognition of bacterial muramyl dipeptide mediated through NOD2. Implications for Crohn's disease. J Biol Chem. 2003; 278: 5509-12.

13. Punzi L, Gava A, Galozzi P, Sfriso P. Miscellaneous non-inflammatory musculoskeletal conditions. Blau syndrome. Best Pract Res Clin Rheumatol. 2011; 25: 703-14.

14. Chamaillard M, Philpott D, Girardin SE, Zouali H, Lesage S, Chareyre F, et al. Geneenvironment interaction modulated by allelic heterogeneity in inflammatory diseases. Proc Natl Acad Sci U S A. 2003; 100: 3455-60.

15. Aróstegui JI, Arnal C, Merino R, Modesto C, Antonia Carballo M, Moreno P, et al. NOD2 gene-associated pediatric granulomatous arthritis: clinical diversity, novel and recurrent mutations, and evidence of clinical improvement with interleukin-1 blockade in a Spanish cohort. Arthritis Rheum. 2007; 56: 3805-13.

16. Martin TM, Zhang Z, Kurz P, Rosé CD, Chen $\mathrm{H}, \mathrm{Lu} \mathrm{H}$, et al. The NOD2 defect in Blau syndrome does not result in excess interleukin-1 activity. Arthritis Rheum. 2009; 60: 611-8.

17. Masumoto J, Yamazaki T, Ohta K, Nakayama J, Agematsu K. Interleukin-1beta suppression in Blau syndrome: comment on the article by Martin et al. Arthritis Rheum. 2009; 60: 2544-5.

18. Son S, Lee J, Woo CW, Kim I, Kye Y, Lee K, et al. Altered cytokine profiles of mononuclear cells after stimulation in a patient with Blau syndrome. Rheumatol Int. 2010; 30: 1121-4.

19. Raphael SA, Blau EB, Zhang WH, Hsu SH. Analysis of a large kindred with Blau syndrome for HLA, autoimmunity, and sarcoidosis. Am J Dis Child. 1993; 147: 842-8.
20. Lachmann HJ, Quartier P, So A, Hawkins PN. The emerging role of interleukin-1in autoinflammatory diseases. Arthritis Rheum. 2011; 63: 314-24.

21. Jéru I, Hentgen V, Normand S, Duquesnoy P, Cochet E, Delwail A, et al. Role of interleukin-1 $\beta$ in NLRP12-associated autoinflammatory disorders and resistance to antiinterleukin-1 therapy. Arthritis Rheum. 2011; 63: 2142-8.

22. Ovadia A, Livneh A, Feld O, Ben-Zvi I, Kukuy E, Kivity S, et al. T helper 17 polarization in familial Mediterranean fever. Genes Immun. 2013; 14: 212-6.

23. Zhou ZY, Chen SL, Shen N, Lu Y. Cytokines and Behcet's disease. Autoimmun Rev. 2012; 11: 699-704.

24. Chen DY, Lan JL, Lin FJ, Hsieh TY. Proinflammatory cytokine profiles in sera and pathological tissues of patients with active untreated adult onset still's disease. J Rheumatol. 2004; 31: 2189-98.

25. Gür-Toy G, Lenk N, Yalcin B. Serum interleukin-8 as a serologic marker of activity in Behçet's disease. Int J Dermatol 2005; 44: 657-60.

26. Choi JH, Suh CH, Lee YM, Suh YJ, Lee SK, Kim SS, et al. Serum cytokine profiles in patients with adult onset Still's disease. J Rheumatol 2003; 30: 2422-7.

27. Nowlan ML, Drewe E, Bulsara H, Esposito N, Robins RA, Tighe PJ, et al. Systemic cytokine levels and the effects of etanercept in TNF receptor-associated periodic syndrome (TRAPS) involving a C33Y mutation in TNFRSF1A. Rheumatology. 2006; 45: 31-7.

28. Jimenez-Martinez MC, Cruz F, GromanLupa S, Zenteno JC. Immunophenotyping in peripheral blood mononuclear cells, aqueous humour and vitreous in a Blau syndrome patient caused by a novel NOD2 mutation. Int $\mathbf{J}$ Immunogenet. 2011; 38: 233-42.

29. Rosenzweig HL, Kawaguchi T, Martin TM, Planck SR, Davey MP, Rosenbaum JT. Nucleotide oligomerization domain-2 (NOD2)induced uveitis: dependence on IFN-gamma. Invest Ophthalmol Vis Sci. 2009; 50: 1739-45. 